\title{
Efeito do fogo na estrutura e estocagem de carbono de um fragmento florestal da mata Atlântica
}

\author{
Bruno Leão Said SCHETTINI ${ }^{1 *}$, Carlos Moreira Miquelino Eleto TORRES ${ }^{1}$, Laércio Antônio Gonçalves \\ JACOVINE ${ }^{1}$, Paulo Henrique VILLANOVA ${ }^{1}$, Samuel José Silva Soares da ROCHA ${ }^{1}$, \\ Maria Paula Miranda Xavier RUFINO ${ }^{1}$, Fillippe Tamiozzo Pereira TORRES ${ }^{1}$ \\ ${ }^{1}$ Departamento de Engenharia Florestal, Universidade Federal de Viçosa, Viçosa, MG, Brasil. \\ E-mail: bruno.schettini@ufv.br
}

Recebido em junho/2018; Aceito em outubro/2018.

\begin{abstract}
RESUMO: O objetivo do trabalho foi avaliar o efeito do fogo na estrutura e estocagem de carbono de uma capoeira, e comparar qual o impacto na vegetação da queima prescrita $(\mathrm{QP})$ em relação ao incêndio não controlado (INC). O estudo foi conduzido em um fragmento florestal em estágio inicial de regeneração de 1,94 ha. INC's foram observados no local entre os anos de 2014 e 2017. Em um desses incêndios, no ano de 2017, parte da capoeira $(0,37 \mathrm{ha})$ foi atingida. $O$ restante da área $(1,57 \mathrm{ha})$ foi submetido ao procedimento de QP, para comparar a diferença entre as duas ações na vegetação. Foi realizado inventário florestal do tipo censo, nos anos de 2013, 2015 e 2017, e, posteriormente, realizadas avaliações de estrutura horizontal, volumetria e estoque de carbono. A densidade absoluta (DA), no ano de 2017 foi $64,37 \%$ inferior ao primeiro levantamento. A dominância absoluta (DoA) foi 74,24\% inferior que no inventário de 2013. Mabea fistulifera Mart. foi a espécie de maior valor de cobertura (VC) nos três inventários. O INC afetou drasticamente a estrutura da floresta. O número de indivíduos ha ${ }^{-1}$ da QP foi 51,86\% superior ao do INC. O impacto do incêndio trouxe danos a estrutura da floresta, bem como ao seu potencial de mitigação das mudanças climáticas enquanto a QP produziu impacto menor.
\end{abstract}

Palavras-chave: degradação florestal, incêndio não controlado, queima prescrita.

\section{Effect of fire on the structure and carbono storage of a forest fragmente of the Atlantic forest}

\begin{abstract}
The aim of this work was to evaluate the effect of fire on the structure and carbon storage of a capoeira, and to compare the impact on the vegetation of the prescribed burning $(\mathrm{QP})$ in relation to uncontrolled fire (INC). The study was conducted in a forest fragment at the initial stage of regeneration of 1.94 ha. INC were observed at the site between the years 2014 and 2017. In one of these fires, in the year 2017, part of the capoeira $(0,37 \mathrm{ha})$ was affected. The rest of the area $(1.57 \mathrm{ha})$ was submitted to the QP procedure, for comparation between the two actions in the vegetation. A census-type forest inventory was carried out in the years 2013, 2015 and 2017 , followed by assessments of horizontal structure, volumetry and carbon stock. The absolute density (AD) in 2017 was $64.37 \%$ lower than the first survey. The absolute dominance (DoA) was $74.24 \%$ lower than in the inventory of 2013. Mabea fistulifera Mart. was the species with the highest coverage value (CV) in all three inventories. The INC affected drastically the structure of the forest. The number of individuals ha ${ }^{-1}$ of QP was $51.86 \%$ higher than that of INC. The impact of the fire has damaged the forest structure as well as its potential to mitigate climate change and QP has a smaller impact on the forest than the INC.
\end{abstract}

Keywords: forest degradation, uncontrolled fire, prescribed burning.

\section{INTRODUÇÃO}

A Mata Atlântica é o bioma brasileiro mais afetado pela fragmentação florestal, e conta com $12,5 \%$ da sua extensão original com remanescentes florestais (INPE, 2014). Um dos motivos para esse quadro de degradação são os incêndios florestais, um dos principais problemas ambientais do mundo moderno (GOBBO et al., 2016). Sua ocorrência pode levar a perdas ecológicas e econômicas consideráveis, bem como culminar em emissões globais de $\mathrm{CO}_{2}$ comparáveis à da queima de combustíveis fósseis (BOWMAN et al., 2009).

Incêndios têm impacto considerável nas condições bióticas e abióticas de um bioma, sendo em alguns ecossistemas, essenciais para o seu funcionamento, como no caso do cerrado onde o fogo representa uma das principais ameaças a sua existência (GOMES et al., 2018). Nas últimas décadas, porém, o aumento significativo nas ocorrências, acarretou na fragmentação e degradação de diferentes ambientes florestais (ADAMEK et al., 2015).

A redução do número de indivíduos é um impacto esperado após um incêndio florestal, devido a mortalidade (LOPES et al., 2009). Porém ocorre o aumento de espécies pioneiras no local, já que elas são favorecidas pela maior incidência de luz, em função das clareiras criadas com a passagem do fogo (XAVIER et al., 2011). Após a ocorrência de um incêndio, com inventários realizados 1,5 e 15 anos após o evento, foi verificada elevada taxa de ingresso, crescimento de indivíduos nos centros de classes superiores, e a substituição espécies pioneiras por espécies clímax, tal fato mostra que mesmo após 
o fogo a vegetação da Mata Atlântica continua a avançar seu estágio sucessional, comprovando sua capacidade de resiliência ao fogo (SILVA, 2017).

O uso do fogo no bioma Mata Atlântica é proibido. Porém, a ocorrência de incêndios florestais é frequente. Apesar disso, é observada regeneração natural em alguns locais, e o efeito do fogo nesse processo necessita de mais estudos (SANTOS, 2017). O uso do fogo pode ser controlado ou não. Incêndio florestal é definido como a propagação livre do fogo. A queima prescrita é a utilização do fogo de maneira controlada, com um determinado objetivo (SOARES; SANTOS, 2002). A proporção do incêndio, bem como seus impactos podem variar em função das interações entre vegetação, clima, topografia e material combustível disponível (TORRES et al., 2017a).

A queima prescrita é uma técnica que, se utilizada, pode amenizar tais impactos na região, uma vez que reduz o material combustível disponível na área (LUCAS-BORJA et al., 2016), diminuindo os riscos dos incêndios de maior intensidade. Além disso, a técnica facilita a regeneração de algumas espécies, em ambientes degradados (SCHWARTZ et al., 2016). O seu uso é bastante difundido, porém existem poucos estudos sobre seu efeito no estoque de carbono acima do solo e na estrutura da floresta.

O fogo é um dos fatores que podem alterar a composição e estrutura de uma floresta, e seus efeitos estão diretamente relacionadas a intensidade e a duração do evento (LOPES et al., 2009). Ter conhecimento do histórico do fogo e de sua área de ocorrência é de extrema importância no auxílio do planejamento de proteção florestal. Compreender o comportamento do fogo e sua resposta às condições ambientais, permite antecipar situações perigosas, amenizar impactos de grande magnitude, e auxiliar na tomada de decisões sobre a gestão de combustíveis e dos incêndios (LINN et al., 2012).

Dessa forma, o objetivo do presente estudo foi avaliar o efeito do fogo na estrutura florestal e estocagem de carbono de uma capoeira, no município de Viçosa-MG e comparar o impacto de um incêndio criminoso e da queima prescrita na vegetação.

\section{MATERIAL E MÉTODOS}

\subsection{Caracterização do local de estudo}

$\mathrm{O}$ estudo foi conduzido em um fragmento florestal em estágio inicial de regeneração (capoeira) de 1,94 ha, localizado no espaço aberto de eventos do campus sede da Universidade Federal de Viçosa $\left(20^{\circ} 45^{\prime} \mathrm{S}\right.$ e $\left.42^{\circ} 52^{\prime} \mathrm{W}\right)$. A área está em processo de regeneração natural, apesar de haver constante intervenção antrópica. Incêndios foram observados no local nos anos de 2014, 2015, 2016 e 2017 (Figura 1).

\subsection{Coleta de dados}

Foi realizado inventário florestal do tipo censo, em que todas as árvores com diâmetro a 1,30 metros de altura do solo (dap) $\geq 5 \mathrm{~cm}$ foram identificadas e tiveram seu dap e altura total (Ht) mensurados. A coleta de dado foi realizada nos anos de 2013, 2015 e 2017. As árvores, após o inventário, foram separadas em centros de classe com amplitude diamétrica de 5 $\mathrm{cm}$.

\subsection{Queima prescrita}

Em um dos incêndios não controlados, ocorrido no ano de 2017, parte da capoeira $(0,37 \mathrm{ha})$ foi atingida. O restante da área $(1,57 \mathrm{ha})$ foi submetido ao procedimento de queima prescrita (QP) para comparação entre as duas ações na estrutura, volumetria, número de indivíduos vivos e estocagem de carbono na vegetação. $\mathrm{O}$ fogo controlado foi utilizado com o objetivo de diminuir o material combustível, como alternativa para tentar minimizar os riscos de possíveis incêndios futuros, dado o histórico do local.

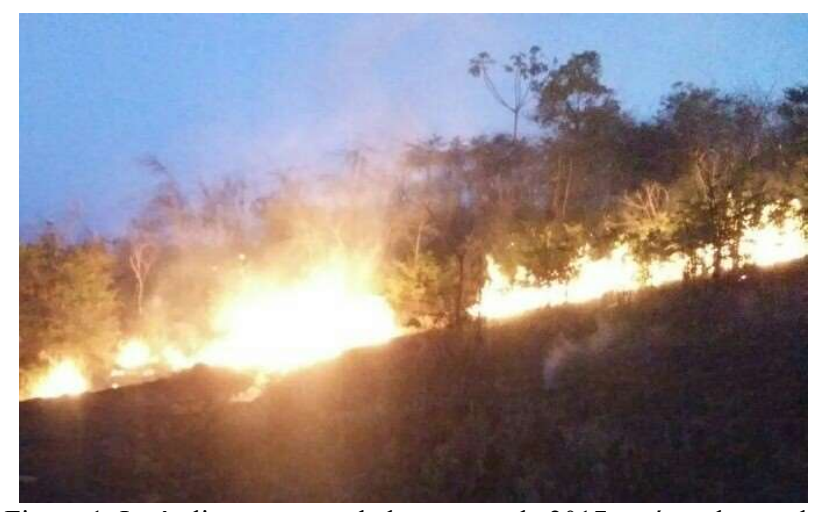

Figura 1. Incêndio não controlado, no ano de 2017 na área de estudo em que foi realizado o inventário florestal.

Figure 1. Uncontrolled fire, in the year 2017 in the study area where the forest inventory was carried out.

\subsection{Estrutura da floresta}

A estrutura horizontal da floresta foi avaliada por meio da densidade absoluta (DAi) e relativa (DRi), dominância absoluta (DoAi) e relativa (DoAi) e Valor de cobertura (VCi).

$$
\begin{array}{ll}
\mathrm{DAi}=\frac{\mathrm{ni}}{\mathrm{A}} & (\text { Equação 1) } \\
\mathrm{DRi}=\frac{\mathrm{ni}}{\mathrm{N}} * 100 & (\text { Equação 2) }
\end{array}
$$

em que: $\mathrm{DA}=$ Densidade absoluta; $\mathrm{DR}=$ Densidade relativa; $\mathrm{ni}=$ número de indivíduos amostrados da i-ésima espécie; $\mathrm{N}=$ número total de indivíduos amostrados; $\mathrm{A}$ = área total amostrada, em hectare (ha).

$$
\begin{array}{ll}
\operatorname{DoAi}=\frac{\sum A S j}{\mathrm{~A}} & (\text { Equação 3) } \\
\mathrm{DoRi}=\frac{\mathrm{DoAi}^{*}}{\sum \mathrm{DoAi}^{*} 100} & \text { (Equação 4) }
\end{array}
$$

em que: DoAi =Dominância absoluta; DoRi $=$ Dominância relativa; $\mathrm{ASi}$ = área seccional ou transversal do tronco ou fuste da j-ésima árvore, da i-ésima espécie; A = área total amostrada, em hectare (ha).

$$
\operatorname{VCi}(\%)=\frac{(\mathrm{DRi}+\mathrm{DoRi})}{2}
$$

em que: $(\mathrm{VCi})=$ Valor de cobertura; $\mathrm{DR}=$ Densidade relativa; DoRi $=$ Dominância relativa.

\subsection{Volume, densidade madeira e estoque de carbono}

O volume de madeira foi estimado baseado na equação do CETEC (1995), para florestas secundárias no estado de Minas Gerais:

$$
\text { VTCC }=0,000074 * \text { dap }^{1,707348} * \mathrm{Ht}^{1,16873} \quad \text { (Equação 6) }
$$

em que: $\mathrm{VTCC}=$ volume total do fuste com casca, em m3; dap = diâmetro a 1,30 metros de altura do solo, em $\mathrm{cm} ; \mathrm{Ht}=$ altura total do indivíduo, em $\mathrm{m}$. 
A biomassa florestal foi obtida pela multiplicação do volume fuste pela densidade básica da madeira. Após a identificação das espécies encontradas na capoeira, foi pesquisada na literatura seus respectivos valores de densidade (BRIANEZI; 2012; TORRES et al., 2017b). O estoque de carbono da madeira do povoamento, nos dois momentos, foi obtido com o uso do fator 0,47 , que é o recomendado para espécies arbóreas (EGGLESTON et al, 2006).

\section{RESULTADOS}

3.1. Distribuição diamétrica

Em todos os inventários o maior número de indivíduos foi observado no centro de classe de $7,5 \mathrm{~cm}$. A distribuição diamétrica em todos os levantamentos tem o comportamento de J-invertido (Figura 2).

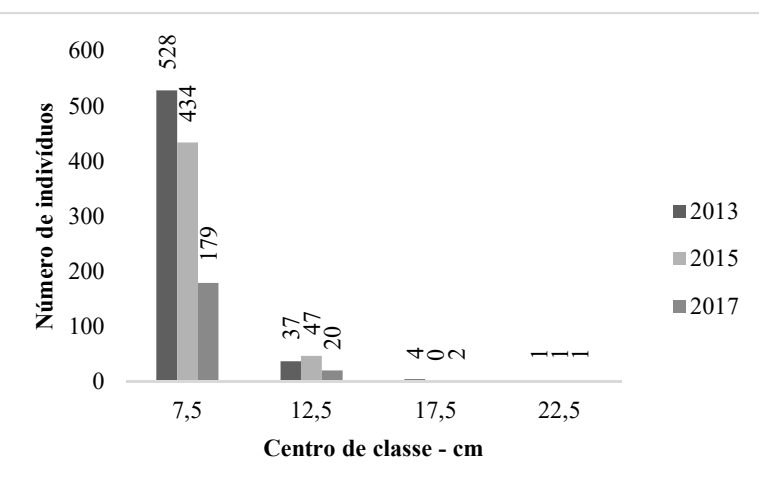

Figura 2. Distribuição diamétrica das árvores inventariadas no fragmento florestal, nos anos de 2013, 2015 e 2017.

Figure 2. Diametric distribution of trees inventoried in the forest fragment, in the years of 2013, 2015 and 2017.

\subsection{Estrutura horizontal}

No primeiro inventário (2013) foram mensurados 567 indivíduos pertencentes a 5 espécies distintas. Mabea fistulifera Mart. (Canudo de Pito) representou 94,00\% do total na área. No segundo inventário (2015), a espécie também foi dominante $(95,69 \%$ do total). Porém, em virtude do incêndio foi mensurado um número menor de indivíduos (482) de 4 espécies diferentes. A partir de 2015 os incêndios se tornaram mais recorrentes no local e após o inventário Mabea fistulifera se manteve como espécie dominante $(95,04 \%$ do total). Porém, o número de indivíduos mensurados (202) foi 41,91\% inferior ao inventário de 2015.

A densidade absoluta (DA) entre 2013 e 2015, reduziu de 292,2680 para 248,4536 árvores ha-1 $(14,99 \%)$ (Tabela 1$)$. No ano de 2017 o efeito do fogo foi mais severo com o total de 104,1237 árvores $\mathrm{ha}^{-1}(64,37 \%$ inferior ao primeiro levantamento). A dominância absoluta (DoA) caiu de 1,9915 para $1,5016 \mathrm{~m}^{2} \mathrm{ha}^{-1}(24,59 \%)$, no período de 2013-2015. No ano de 2017 a DoA foi $74,24 \%$ inferior que no primeiro levantamento $\left(0,5129 \mathrm{~m}^{2} \mathrm{ha}^{-1}\right)$. A Mabea fistulifera foi a espécie de maior valor de cobertura (VC) nos três inventários. Em 2013 o segundo maior VC foi da Cecropia hololeuca Miq. (Embaúba) porém em 2015 Anadenanthera macrocarpa Benth e Brenan (Angico Vermelho). As espécies Caesalpinia peltophoroides Benth. (Sibipiruna) e Luehea grandiflora Benth e Zucc (Açoita Cavalo) não foram observadas no segundo inventário, e o Inga sp. (Ingá) foi mensurado apenas em 2015. No ano de 2017 foram inventariadas as mesmas espécies do inventário de 2015.

3.3. Volume, densidade madeira, biomassa e estoque de carbono

$\mathrm{O}$ volume de madeira, ao final do terceiro inventário, reduziu 44,91\%. O acúmulo de biomassa e estoque de carbono, findados os levantamentos, foram 44,58 e 44,53\% inferiores, respectivamente, ao encontrado em 2013. A espécie com maior volume e estoque de carbono na madeira foi a Mabea fistulifera (Tabela 2).

\subsection{Queima prescrita $x$ incêndio não controlado}

O incêndio não controlado afetou drasticamente a estrutura da floresta. O número de indivíduos $\mathrm{ha}^{-1}$ presentes na área sujeita a QP foi 51,86\% superior ao do INC. A DoA da QP foi $68,25 \%$ superior ao do INC (Tabela 3).

O volume de madeira no local onde ocorreu a QP foi $75,57 \%$ superior ao de ocorrência do INC. Os valores de acúmulo de biomassa e estoque de carbono também foram superiores $(73,02 \%)$. A riqueza de espécies da área em que houve a QP foi superior ao INC (Tabela 3).

Tabela 1. Estrutura horizontal do fragmento florestal avaliado nos anos de 2013, 2015 e 2017

Table 1. Horizontal structure of the forest fragment evaluated in the years of 2013,2015 and 2017

\begin{tabular}{|c|c|c|c|c|c|c|}
\hline Ano & Espécie & $\begin{array}{c}\text { DA } \\
\left(\mathrm{n} \mathrm{ha}^{-1}\right) \\
\end{array}$ & $\begin{array}{l}\text { DR } \\
(\%) \\
\end{array}$ & $\begin{array}{c}\text { DoA } \\
\left(\mathrm{m}^{2} \mathrm{ha}^{-1}\right)\end{array}$ & $\begin{array}{c}\text { DoR } \\
(\%) \\
\end{array}$ & $\begin{array}{l}\mathrm{VC} \\
(\%) \\
\end{array}$ \\
\hline \multirow{6}{*}{2013} & Mabea fistulifera Mart. & 274,7423 & 94,00 & 1,8352 & 92,15 & 93,08 \\
\hline & Cecropia hololeuca Miq. & 4,1237 & 1,41 & 0,1024 & 5,14 & 3,28 \\
\hline & Luehea grandiflora Benth e Zucc & 11,8557 & 4,06 & 0,0448 & 2,25 & 3,15 \\
\hline & Anadenanthera macrocarpa Benth e Brenan & 1,0309 & 0,35 & 0,0071 & 0,36 & 0,36 \\
\hline & Caesalpinia peltophoroides Benth. & 0,5155 & 0,18 & 0,0019 & 0,10 & 0,14 \\
\hline & Total & 292,2680 & 100 & 1,9915 & 100 & 100 \\
\hline Ano & Espécie & $\mathrm{DA}$ & DR & DoA & DoR & $\mathrm{VC}$ \\
\hline \multirow{5}{*}{2015} & Mabea fistulifera Mart. & 245,3608 & 98,76 & 1,4721 & 98,03 & 98,40 \\
\hline & Anadenanthera macrocarpa Benth e Brenan & 1,5464 & 0,62 & 0,0075 & 0,50 & 0,56 \\
\hline & Cecropia hololeuca Miq. & 1,0309 & 0,41 & 0,0208 & 1,38 & 0,90 \\
\hline & Inga sp & 0,5155 & 0,21 & 0,0012 & 0,08 & 0,14 \\
\hline & Total & 248,4536 & 100 & 1,5016 & 100 & 100 \\
\hline Ano & Espécie & $\mathrm{DA}$ & DR & DoA & DoR & $\mathrm{VC}$ \\
\hline \multirow{4}{*}{2017} & Mabea fistulifera Mart. & 98,9690 & 95,05 & 0,4667 & 0,9099 & 0,9302 \\
\hline & Inga sp & 4,1237 & 3,95 & 0,0193 & 0,0377 & 0,0387 \\
\hline & Cecropia hololeuca Miq. & 0,5155 & 0,50 & 0,0255 & 0,0498 & 0,0274 \\
\hline & Anadenanthera macrocarpa Benth e Brenan & 0,5155 & 0,50 & 0,0013 & 0,0026 & 0,0038 \\
\hline
\end{tabular}

Nativa, Sinop, v. 7, n. 3, p. 306-311, mai/jun. 2019. 
Total

104,1237

100

0,5129

100

100

Tabela 2. Volume, densidade básica da madeira, acúmulo de biomassa e estoque de carbono das espécies observadas na capoeira nos anos de 2013, 2015 e 2017

Table 2. Volume, basic wood density, biomass accumulation and carbon stock of the species observed in capoeira in the years of 2013,2015 and 2017

\begin{tabular}{|c|c|c|c|c|c|}
\hline Ano & Espécie & Volume $\left(\mathrm{m}^{3} \mathrm{ha}^{-1}\right)$ & Densidade $\left(\mathrm{g} \mathrm{cm}^{-3}\right)$ & Biomassa $\left(\mathrm{MgC} \mathrm{ha}^{-1}\right)$ & Carbono $\left(\mathrm{MgC} \mathrm{ha}^{-1}\right)$ \\
\hline \multirow{6}{*}{2013} & Mabea fistulifera Mart. & 7,1629 & 0,5580 & 3,9969 & 1,8785 \\
\hline & Cecropia hololeuca Miq. & 0,5665 & 0,3720 & 0,2107 & 0,0990 \\
\hline & Luehea grandiflora Benth e Zucc & 0,0943 & 0,4900 & 0,0462 & 0,0217 \\
\hline & Anadenanthera macrocarpa Benth e Brenan & 0,0195 & 0,5540 & 0,0108 & 0,0051 \\
\hline & Caesalpinia peltophoroides Benth. & 0,0081 & 0,7343 & 0,0059 & 0,0028 \\
\hline & Total & 7,8513 & - & 4,2706 & 2,0072 \\
\hline Ano & Espécie & Volume $\left(\mathrm{m}^{3} \mathrm{ha}^{-1}\right)$ & Densidade $\left(\mathrm{g} \mathrm{cm}^{-3}\right)$ & Biomassa $\left(\mathrm{MgC} \mathrm{ha}^{-1}\right)$ & Carbono $\left(\mathrm{MgC} \mathrm{ha}^{-1}\right)$ \\
\hline \multirow{5}{*}{2015} & Mabea fistulifera Mart. & 6,6333 & 0,5580 & 3,7014 & 1,7397 \\
\hline & Cecropia hololeuca Miq. & 0,0260 & 0,3720 & 0,0097 & 0,0045 \\
\hline & Anadenanthera macrocarpa Benth e Brenan & 0,0197 & 0,5540 & 0,0109 & 0,0051 \\
\hline & Inga sp & 0,0025 & 0,4971 & 0,0012 & 0,0006 \\
\hline & Total & 6,6815 & - & 3,7232 & 1,7499 \\
\hline Ano & Espécie & Volume $\left(\mathrm{m}^{3} \mathrm{ha}^{-1}\right)$ & Densidade $\left(\mathrm{g} \mathrm{cm}^{-3}\right)$ & Biomassa $\left(\mathrm{MgC} \mathrm{ha}^{-1}\right)$ & Carbono $\left(\mathrm{MgC} \mathrm{ha}^{-1}\right)$ \\
\hline \multirow{5}{*}{2017} & Mabea fistulifera Mart. & 4,0136 & 0,5580 & 2,2396 & 1,0536 \\
\hline & Cecropia hololeuca Miq. & 0,2219 & 0,3720 & 0,0825 & 0,0388 \\
\hline & Inga sp & 0,0851 & 0,4971 & 0,0423 & 0,0199 \\
\hline & Anadenanthera macrocarpa Benth e Brenan & 0,0043 & 0,5540 & 0,0024 & 0,0011 \\
\hline & Total & 4,3249 & - & 2,3668 & 1,1134 \\
\hline
\end{tabular}

Tabela 3. Análise comparativa da estrutura horizontal, volume, biomassa e estoque de carbono no fragmento florestal avaliado entre as áreas de ocorrência do incêndio não controlado (INC) e da queima prescrita (QP)

Table 3. Comparative analysis of the horizontal structure, volume, biomass and carbon stock in the forest fragment assessed between the areas of occurrence of uncontrolled fire (INC) and prescribed burning (QP)

\begin{tabular}{|c|c|c|c|c|c|c|c|c|c|}
\hline Avaliação & Espécie & $\begin{array}{c}\text { DA } \\
\left(\mathrm{n} \mathrm{ha}^{-1}\right)\end{array}$ & $\begin{array}{l}\text { DR } \\
(\%)\end{array}$ & $\begin{array}{c}\text { DoA } \\
\left(\mathrm{m}^{2} \mathrm{ha}^{-1}\right)\end{array}$ & $\begin{array}{l}\text { DoR } \\
(\%)\end{array}$ & $\begin{array}{l}\mathrm{VC} \\
(\%)\end{array}$ & $\begin{array}{l}\text { Volume } \\
\left(\mathrm{m}^{3} \mathrm{ha}^{-1}\right)\end{array}$ & $\begin{array}{l}\text { Biomassa } \\
\left(\mathrm{MgC} \mathrm{ha}^{-1}\right)\end{array}$ & $\begin{array}{l}\text { Carbono } \\
\left(\mathrm{MgC} \mathrm{ha}^{-1}\right)\end{array}$ \\
\hline $\mathrm{INC}$ & Mabea fistulifera Mart. & 59,4595 & 100 & 0,3220 & 100 & 100 & 1,3296 & 0,7419 & 0,3487 \\
\hline & Total & 59,4595 & 100 & 0,3220 & 100 & 100 & 1,3296 & 0,7419 & 0,3487 \\
\hline Ano & Espécie & $\begin{array}{c}\text { DA } \\
\left(\mathrm{n} \mathrm{ha}^{-1}\right)\end{array}$ & $\begin{array}{l}\text { DR } \\
(\%)\end{array}$ & $\begin{array}{c}\text { DoA } \\
\left(\mathrm{m}^{2} \mathrm{ha}^{-1}\right)\end{array}$ & $\begin{array}{l}\text { DoR } \\
(\%)\end{array}$ & $\begin{array}{l}\mathrm{VC} \\
(\%)\end{array}$ & $\begin{array}{l}\text { Volume } \\
\left(\mathrm{m}^{3} \mathrm{ha}^{-1}\right)\end{array}$ & $\begin{array}{l}\text { Biomassa } \\
\left(\mathrm{MgC} \mathrm{ha}^{-1}\right)\end{array}$ & $\begin{array}{l}\text { Carbono } \\
\left(\mathrm{MgC} \mathrm{ha}^{-1}\right)\end{array}$ \\
\hline \multirow{5}{*}{$\mathrm{QP}$} & Mabea fistulifera Mart. & 108,28 & 94,46 & 0,9536 & 94,02 & 94,2 & 4,6461 & 2,5925 & 1,2185 \\
\hline & Inga sp & 5,0955 & 4,44 & 0,0275 & 2,71 & 3,58 & 0,2742 & 0,1020 & 0,0480 \\
\hline & Cecropia hololeuca Miq. & 0,6369 & 0,55 & 0,0316 & 3,11 & 1,83 & 0,1052 & 0,0523 & 0,0246 \\
\hline & Anadenanthera macrocarpa Benth e Brenan & 0,6369 & 0,55 & 0,0016 & 0,16 & 0,36 & 0,0053 & 0,0030 & 0,0014 \\
\hline & Total & 114,65 & 100 & 1,0143 & 100 & 100 & 5,0308 & 2,7498 & 1,2925 \\
\hline
\end{tabular}

\section{DISCUSSÃO}

O censo realizado permitiu a comparação entre as três medições $(2013,2015$ e 2017), e os resultados mostraram uma perda significativa em área basal $(55,70 \%)$, densidade de plantas, volume, biomassa e carbono, mesmo sendo quantificadas as árvores ingressantes.

A redução do número de indivíduos e espécies também foi observada por Lima et al. (2009), que ao avaliarem o efeito do fogo em uma vegetação, no período de 5 anos, concluíram que a intensidade do fogo pode remover espécies de um fragmento florestal. Friedler et al. (2004) ao avaliar o efeito do fogo em uma área de cerrado observaram, diferentemente do presente estudo, o aumento da densidade de indivíduos por hectare. Essa diferença, provavelmente, decorre do método de inventário utilizado, já que foram mensurados todos os indivíduos com diâmetro a $30 \mathrm{~cm}$ de altura $\geq 3 \mathrm{~cm}$. Além disso, o cerrado é um bioma com grande potencial de regeneração natural e adaptado ao fogo (ALVARADO et al., 2017). Ao avaliar o efeito de um incêndio florestal, a redução da biomassa e número de espécies é uma consequência esperada, em decorrência da morte parcial ou total dos indivíduos (SILVA et al., 2005). Assim como no presente estudo, Lopes et al. (2009) observaram a redução do número de indivíduos e espécies nos diferentes centros de classe diamétrica avaliados, em função da intensidade do fogo, que impacta mais os centros de classe inferiores.

Nos inventários, Mabea fistulifera foi a espécie dominante, representando $91,23 \%, 99,28 \%$ e $92,80 \%$ do volume total em 2013, 2015 e 2017, respectivamente. A espécie se enquadra no grupo ecológico das pioneiras e é constantemente observada em áreas antropizadas na Mata Atlântica (CALGARO et al., 2015). O número maior de indivíduos da espécie pode ser explicado possivelmente pela sua associação com formigas. No caso da espécie, as formigas possuem taxa de remoção de sementes com variação de 85 a 97 \%, que justificam o maior número de indivíduos da Mabea fistulifera no local, uma vez que transportam efetivamente as sementes favorecendo sua dispersão (PETERNELLI et al., 2004).

As florestas possuem potencial de mitigar as alterações no clima, pois estocam carbono na forma de biomassa florestal (DAVID et al., 2017), e com a redução do volume de madeira esse potencial fica comprometido. Os incêndios causam efeito contrário, uma vez que aumentam as emissões de gases de efeito estufa (GEE) (NING et al., 2017). Foi possível constatar tal fato no presente estudo, uma vez que o volume de madeira 
no período pós fogo foi inferior, e consequentemente reduziu o estoque de carbono.

Os incêndios antropogênicos não controlados degradaram grandes áreas de florestas tropicais nas últimas décadas (BALCH et al., 2011). Uma forma de minimizar seus riscos e impactos é o uso da queima prescrita, que diminui o material combustível na área (MOLINA et al., 2018), reduzindo a intensidade e a gravidade dos incêndios não controlados (MATSYPURA et al., 2018). Tal fato foi observado no presente estudo, uma vez que a densidade e dominância, bem como o volume, biomassa e estoque de carbono, foram superiores na área sujeita a queima prescrita, quando comparada aquela onde ocorreu o incêndio. Os incêndios florestais de alta severidade devem ser evitados, pois comprometem o ecossistema e acentuam o efeito das alterações no clima. Além disso, criam condições que dificultam a regeneração do local e o reestabelecimento da florestal (TABOADA et al., 2017).

\section{CONCLUSÕES}

Mabea fistulifera foi a espécie dominante nos dois inventários.

Os incêndios florestais não controlados, quando comparados ao procedimento de queima prescrita, afetam de maneira mais drástica os valores de dominância, densidade, frequência e estoque de carbono de uma floresta.

O impacto do incêndio trouxe danos a estrutura da floresta, bem como ao seu potencial de mitigação das mudanças climáticas.

A queima prescrita apresentou impacto menor na floresta do que o incêndio não controlado.

\section{REFERÊNCIAS}

ADAMEK, M.; BOBEK, P.; HADINCOVÁ, V.; WILD, J.; KOPECKY, M. Forest fires within a temperate landscape: A decadal and millennial perspective from a sandstone region in Central Europe. Forest Ecology and Management, Amsterdam, v. 336, p. 81-90, 2015. DOI: https://doi.org/10.1016/j.foreco.2014.10.014

ALVARADO, S. T.; FORNAZARI, T.; CÓSTOLA, A.; MORELLATO, L. P. C.; SILVA, T. S. F. Drivers of fire occurrence in a mountainous Brazilian cerrado savanna: Tracking long-term fire regimes using remote sensing. Ecological Indicators, v. 78, n. 1, p. 270 - 281, 2017. DOI: https://dxdoi.org/10.1016/j.ecolind.2017.02.037

BALCH, J. K.; NEPSTAD, D. C.; CURRAN, L. M.; BRANDO, P. M.; PORTELA, O.; GUILHERME, P.; REUNING-SCHERER, J. D.; CARVALHO JR, W. Size, species, and fire behavior predict tree and liana mortality from experimental burns in the Brazilian Amazon. Forest Ecology and Management, Amsterdam, v. 261, n. 1, p. 68-77, 2011.

DOI: https://doi.org/10.1016/j.foreco.2010.09.029

BOWMAN, D. M. S. J.; BALCH, J. K.; ARTAXO, P.; BOND, W. J.; CARLSON, J. M.; COCHRANE, M. A.; D’ANTONIO, C. M.; DEFRIES, R. S.; DOYLE, J. C.; HARRISON, S. P.; JOHNSTON, F. H.; KEELEY, J. E.; KRAWCHUK, M. A.; KULL, C. A.; MARSTON, J. B.; MORITZ, M. A.; PRENTICE, I. C.; ROOS, C. I.; SCOTT, A. C.; SWETNAM, T. W.; VAN DER WEF, G. R.; PYNE, S. J. Fire in the Earth System. Science, Washington, v. 324, n. 1 , p. $481 \quad-\quad 484, \quad 2009$. DOI: http://dx.doi.org/10.1126/science. 1163886
BRIANEZI, D. Estocagem e compensação de carbono pelas árvores do campus-sede da Universidade Federal de Viçosa. 2012. 159f. Dissertação (Mestrado em Ciência Florestal) - Universidade Federal de Viçosa, Viçosa, 2012.

CALGARO, H. F.; BUZETTI, S.; SILVA, L. R.; STEFANINI, L.; MIRANDA, L. P. M.; MORAES, M. A.; MORAES, M. L. T. Distribuição natural de espécies arbóreas em áreas com diferentes níveis de antropização e relação com os atributos químicos do solo. Revista Árvore, Viçisa, v. 39, n. 2, p. 233 - 243, 2015. DOI: http://dx.doi.org/10.1590/0100-67622015000200003.

CETEC_FUNDAÇÃO CENTRO TECNOLÓGICO DE MINAS GERAIS. Determinação de equações volumétricas aplicáveis ao manejo sustentado de florestas nativas no estado de Minas Gerais e outras regiões do país. Belo Horizonte: SAT/CETEC, 1995. 295 $\mathrm{p}$.

DAVID, H. C.; ARAÚJO, E. J. G.; MORAIS, V. A.; SCOLFORO, J. R. S.; MARQUES, J. M.; NETTO, S. P.; MACFARLANE, D. W. Carbon stock classification for tropical forests in Brazil: Understanding the effect of stand and climate variables. Forest Ecology and Management, Amsterdam, v. 404, n. 1, p. 241-250, 2017. DOI: https://dx.doi.org/10.1016/j.foreco.2017.08.044

EGGLESTON, H.S.; BUENDIA, L.; MIWA, K.; NGARA, T.; TANABE, K. 2006 IPCC Guidelines for National Greenhouse Gas Inventories. Hayama: IPCC - IGES, 2006. 12 p.

FRIEDLER, N. C.; AZEVEDO, I. N. C.; REZENDE, A. V.; MEDEIROS, M. B.; VENTUROILI, F. Efeito de incêndios florestais na estrutura e composição florística de uma área de cerrado sensu stricto na fazenda Água Limpa - DF. Revista Árvore, Viçosa, v. 28, n. 1, p. 121 - 138, 2004. DOI: http://dx.doi.org/10.1590/S010067622004000100017

GOBBO, S. D. A.; GARCIA, R. F.; AMARAL, A. A.; EUGENIO, F. C.; ALVAREZ, C. R. S.; LUPPI, A. S. L. Uso da terra no entorno do PARNA-Caparaó: preocupação com incêndios florestais. Revista Floresta e Ambiente, Seropédica, v. 23, n. 3, p. 350-361, 2016. DOI: http://dx.doi.org/10.1590/2179-8087.110114

GOMES, L.; MIRANDA, H. S.; BUSTAMENTE, M. M. C. How can we advance the knowledge on the behavior and effects of fire in the Cerrado biome? Forest Ecology and Management, Amsterdam, v. 417, n. 1, p. 281 - 290, 2018. DOI: https://doi.org/10.1016/j.foreco.2018.02.032

INPE_INSTITUTO NACIONAL DE PESQUISAS ESPACIAIS. SOS Mata Atlântica e INPE apresentam dados do Atlas dos Remanescentes Florestais da Mata Atlântica, 2014. Disponível em: $<$ http://www.inpe.br/noticias/noticia.php?Cod_Noticia=3 610>. Acesso em 28 de Novembro de 2017.

LIMA, E. S.; LIMA, H. S.; RATTER, J. A. Mudanças pósfogo na estrutura e composição da vegetação lenhosa, em um cerrado mesotrófico, no período de cinco anos (19972002) em nova xavantina - MT. Cerne, Lavras, v. 15, n. 4, p. $468-480,2009$.

LINN, R. R.; CANFIELD, J. M.; CUNNINGHAM, P.; EDMINSTER, C.; DUPUY, J. L.; PIMONT, F. Using periodic line fires to gain a new perspective on multidimensional aspects of forward fire spread. Agricultural and Forest Meteorology, v. 157, n. 1, p. 60 - 76, 2012. DOI: https://doi.org/10.1016/j.agrformet.2012.01.014 
LOPES, S. F.; DO VALE, V. S.; SCHIAVINI, I. Efeito de queimadas sobre a estrutura e composição da Comunidade vegetal lenhosa do cerrado sentido restrito em Caldas novas, GO. Revista Árvore, Viçosa, v. 33, n. 4, p. 695 704, 2009. DOI: http://dx.doi.org/10.1590/S010067622009000400012

LUCAS-BORJA, M. E.; MADRIGAL, J.; CANDEL-PÉREZ, D.; JIMÉNEZ, E.; MOYA, D.; DE LAS HERAS, J.; GUIJARRO, M.; VEGA, J. A.; FERNÁNDEZ, C.; HERNANDO, C. Effects of prescribed burning, vegetation treatment and seed predation on natural regeneration of Spanish black pine (Pinus nigra Arn. ssp. salzmannii) in pure and mixed forest stands. Forest Ecology and Management, Amsterdam, v. 378, n. 1, p. 24-30, 2016. DOI: https://dx.doi.org/10.1016/j.foreco.2016.07.019

MATSYPURAA, D.; PROKOPYEVB, O. A.; ZAHARA, A. Wildfire fuel management: network-based models and optimization of prescribed burning. European Journal of Operational Research, Amsterdam, v. 264, n. 1, p. 774 796, 2018.

MOLINA, J. R.; GARCÍA, J. P.; FERNÁNDEZ, J. J.; RODRÍGUEZ Y SILVA, F. Prescribed fire experiences on crop residue removal for biomass exploitations. Application to the maritime pine forests in the Mediterranean Basin. Science of The Total Environment, Amsterdam, v. 612, n. 1, p. 63-70, 2018. DOI: https://doi.org/10.1016/j.scitotenv.2017.08.182

NING, Z.; SUN, C. Forest management with wildfire risk, prescribed burning and diverse carbon policies. Forest Policy and Economics, Amsterdam, v. 75, n. 1, p. 95-102, 2017.

PETERNELLI, E. F. O.; LUCIA, T. M. C; MARTINS, S. V. Espécies de formigas que interagem com as sementes de Mabea fistulifera Mart. (Euphorbiaceae). Revista Árvore, Viçosa, v. 28, n. 5, p. 733 - 738, 2004. DOI: http://dx.doi.org/10.1590/S0100-67622004000500013

SANTOS, J. F. C. Dinâmica florestal e detecção de ocorrências do fogo em área do domínio de Mata Atlântica. 2017. 112f. Tese (Doutorado em Engenharia Florestal) - Universidade Federal de Viçosa, Viçosa, 2017.

SCHWARTZ, N. B.; URBAN, D. L.; WHITE, P. S.; MOODY, A.; KLEIN, R. N. Vegetation dynamics vary across topographic and fire severity gradients following prescribed burning in Great Smoky Mountains National Park. Forest Ecology and Management, Amsterdam, v.
365, n. $1, \quad$ p. $1-11, \quad 2016$. DOI: https://doi.org/10.1016/j.foreco.2016.01.027

SILVA, K. G. da. Efeito do fogo na organização espacial e dinâmica em uma floresta tropical. 2017. 102f. Tese (Doutorado em Engenharia Florestal) - Universidade Federal de Lavras, Lavras, 2017.

SILVA, V. F.; OLIVEIRA FILHO, A. T.; VENTURIN, N.; CARVALHO, W. A. C.; GOMES, J. B. V. Impacto do fogo no componente arbóreo de uma floresta estacional semidecídua no município de Ibituruna, MG, Brasil. Acta Botanica Brasilica, São Paulo, v. 19, n. 1, p. 701-716, $2005 . \quad$ DOI: $\quad$ http://dx.doi.org/10.1590/S010233062005000400005

SOARES, R.V.; SANTOS, J.F. Perfil dos incêndios florestais no Brasil de 1994 a 1997. Floresta, Curitiba, v. 32, n. 2, p. $219 \quad-\quad 225, \quad 2002 . \quad$ DOI: http://dx.doi.org/10.5380/rf.v32i2.2287

TABOADA, A.; TÁRREGA, R.; MARCOS, E.; VALBUENA, L.; SUÁREZ-SEOANE, S.; CALVO, L. Fire recurrence and emergency post-fire management influence seedling recruitment and growth by altering plant interactions in fire-prone ecosystems. Forest Ecology and Management, Amsterdam, v. 402, n. 1, p. 63-75, 2017.2 DOI: https://dx.doi.org/10.1016/j.foreco.2017.07.029

TORRES, C. M. M. E.; JACOVINE, L. A. G.; OLIVEIRA NETO, S. N.; SOUZA, A. L.; CAMPOS, R. A.; SCHETTINI, B. L. S. Análise fitossociológica e valor de importância em carbono para uma floresta estacional semidecidual. Floresta e Ambiente, Seropédica, v. 24, n. 1, p. 1 - 10, 2017b. DOI: http://dx.doi.org/10.1590/21798087.099714

TORRES, F. T. P.; ROQUE, M. P. B.; LIMA, G. S.; MARTINS, S. V. M.; FARIA, A. L. L. Mapeamento do risco de incêndios florestais utilizando técnicas de geoprocessamento. Floresta e Ambiente, Seropédica, v. 24 , n. $1, \quad$ p. $1-10, \quad 2017$ a. DOI: http://dx.doi.org/10.1590/2179-8087.025615

XAVIER, K. R. F.; ANDRADE, L. A.; FABRICANTE, J. R.; COELHO, M. S. E.; ASSIS, F. N. M. Impactos pós-fogo na regeneração natural em um fragmento de floresta ombrófila aberta no município de Areia, Paraíba, Brasil. Revista Brasileira de Biosciências, Porto Alegre, v. 9, n. 3, p. $257-264,2011$. 